\title{
Calldad de vida laboral y compromiso organizacional del personal nombrado en la Municipalidad Provincial de San Román
}

\author{
Quality of work life and organizational commitment of personnel appointed in the \\ Provincial Municipality of San Román
}

\author{
Ruth Joella Zavala Cuentas, Luís Eduardo Córdova Carranza \\ ${ }^{1}$ EP. Administración, Facultad de Ciencias Empresariales, Universidad Peruana Unión
}

INFORMACIÓN DEL ARTÍCULO

Historia del artículo

Recibido: agosto de 2017

Aceptado: setiembre de

2018

Palabras clave:

Calidad de vida laboral, compromiso organizacional.

\section{Resumen}

La presente investigación estableció como objetivo, determinar la relación existente entre la Calidad de Vida Laboral y el Compromiso Organizacional del personal nombrado en la sede administrativa de la Municipalidad Provincial de San Román. La metodología utilizada fue de tipo descriptivo correlacional de corte transversal. El tamaño de la muestra fue de 42 servidores públicos nombrados, a los cuales se le aplicó un cuestionario estructurado en tres partes, la primera identificaba los Aspectos Personales y Profesionales, la segunda constituida por la Escala de Calidad de Vida Profesional CVP-35 y por último el Instrumento de Compromiso organizacional. En lo que respecta a los resultados se tiene que los datos obtenidos fueron analizados estadísticamente con la prueba Tau- b de Kendall, cuyas deducciones evidencian que, no existe relación estadísticamente significativa entre la Calidad de Vida Laboral y el Compromiso Organizacional; ni entre la Motivación intrínseca y el Compromiso Organizacional, y el Apoyo directivo con el Compromiso Organizacional. No obstante, sí existen evidencias suficientes que confirman la correlación directa y estadísticamente significativa entre las Cargas del trabajo y el Compromiso Organizacional. Finalmente, las dimensiones que componen la Calidad de Vida Laboral en una institución pública, no influyen de manera relevante en el compromiso que el personal nombrado tiene hacia su institución, pues ellos otorgan mayor importancia a otros aspectos que determinan su compromiso, como el logro de la estabilidad laboral a través del nombramiento, más que a la Calidad de Vida Laboral.

\section{Abstract}

This investigation established the following objectives: to determine the relationship between the quality of working life and organizational commitment of the staff appointed at the administrative headquarters of the Provincial Municipality of San Roman. Method: this is a descriptive correlational study of crosssection. A questionnaire which has been structured in three parts was applied to a sample size made up of 42 officials appointed; the first part identified personal and professional aspects, the second one involved the Scale of Professional Life Quality CVP-35 and finally, the instrument Organizational commitment. Results: The obtained data were statistically analyzed with Tau-b of Kendall test, and the results showed that there is no significant relationship between the quality of working life and organizational commitment; or between intrinsic motivation and organizational commitment; and 
Keywords:

Quality of Working Life, Organizational commitment. also, the management support with organizational commitment However, if there is enough evidence to confirm the direct and significant correlation between the loads of work and organizational commitment. Conclusions: dimensions that make up the Quality of Working Life in a public institution do not influence relevantly on the commitment that the appointed staff has towards their institution, due to they give more importance to other aspects that determine their commitment, as achieving labor stability through the appointment, rather than the Quality of Working Life.

\section{Introducción}

A finales del siglo XVIII un hito que marcó la historia y que trajo muchos cambios en el trabajo fue la Revolución Industrial, en la cual se crearon grandes empresas, por ende, se contrataron millares de trabajadores, quienes representaban solo la mano de obra; eso trajo la organización de los sindicatos para que los representaran y pudieran aminorar la presión constante del control capitalista. Es allí, cuando surgen los postulados de Frederick Taylor, padre de la Administración científica, fue quien señaló la importancia de mantener a los empleados satisfechos en sus puestos laborales, pues hasta ese entonces existía un proceso de deshumanización del trabajo, por ello se tuvo un primer interés que fue el de la racionalización del trabajo que dio pie al conocido estudio de tiempos y movimientos que ejecutaban los operarios en sus tareas a fin de eliminar los desperdicios e incrementar la productividad.

Stoner y colbs (1996), señalan que Taylor "sugirió a los patrones que les pagaran a los trabajadores más productivos una cantidad superior a la de los demás, usando una tasa científicamente correcta, con lo que beneficiaria tanto a la empresa como el trabajador. Así, se fomentaría que los trabajadores superaran los parámetros de sus resultados anteriores, con miras a obtener un mejor sueldo" (p.36).
Esto fue denominado por Taylor como el Sistema de tasas diferenciales de esta manera, nació el interés en el bienestar de los trabajadores que es el objetivo de la calidad de vida laboral, a esto se sumaron estudios posteriores encabezados por Henry Fayol.

Ahora bien, con el transcurrir del tiempo, el enfoque hacia las personas fue más notorio, y en el año 1932 surgió la Escuela de las Relaciones humanas, en la cual se analizó el trabajo para los grupos informales existentes en las empresas, la toma de decisiones y estilos de liderazgo. Gran parte de autores en esta área coinciden en que fue en este momento que se inició el interés por la CVL como tal, dicho suceso fue reforzado con el surgimiento de la teoría del comportamiento en el año 1940.

Más adelante, y a partir de los años 60 ya se empieza a reconocer el valor del comportamiento de los colaboradores dentro del ámbito laboral y se admite la relación de codependencia entre la empresa y los trabajadores, dicha realidad se ha ido reafirmando con los años.

A propósito de ello, Chiavenato (2009), acota al respecto, que las personas de una empresa deben ser tratadas como socios, seres humanos dotados de personalidad propia, con conocimientos, habilidades, destrezas y capacidades indispensa- 
bles para la gestión adecuada de los recursos organizacionales; en consecuencia las personas empiezan a ser consideradas como uno de los factores más importantes de la empresa.

Por otro lado, los trabajadores se consideran sumamente importantes gracias al trabajo que tienen en las empresas, pues gran parte de la vida -treinta a treinta y cinco años aproximadamente, en jornadas de ocho horas diarias- transcurre en el trabajo y es allí donde satisfacen gran parte de sus necesidades. En este contexto, surgieron varios métodos, enfoques y teorías comportamentales y de gestión que buscaron hacer del trabajo una actividad satisfactoria y de bienestar, que a su vez tenga efecto en un alto nivel de productividad, en dicho contexto es donde surge el Compromiso Organizacional y la Calidad de Vida Laboral.

No obstante, en la realidad peruana aún hay mucho por hacer a nivel de gestión del talento humano, gran parte de empresas privadas aun no asignan el valor correspondiente a sus colaboradores y este fenómeno es más complejo en las instituciones públicas. Ugarte, Celle y Sotomarino (2011), afirman que la administración pública no tiene una clara preocupación por sus colaboradores, más bien se presentan ascensos fraguados, ausencia de políticas remunerativas que provocan desorden, abuso y desincentivo a los buenos trabajadores, falta de información, ausencia de políticas sobre programas de capacitación, exceso de sanciones y deficientes sistemas de control de gestión. Todo ello nos demuestra que la administración pública tiene cierto desinterés respecto al bienestar de los servidores públicos. Lo cierto es que, como asevera Reyes (2009), en el Estado al igual que en toda organización, si las personas no se encuentran motivadas, capacitadas, evaluadas, bien remuneradas no se puede esperar un rendimiento óptimo y un servicio de calidad en beneficio de los ciudadanos.

De lo anteriormente expuesto, surge el interés investigativo de conocer la realidad respecto a la Calidad de Vida Laboral (CVL), y el Compromiso Organizacional (CO), en una institución pública a fin de conocer si existe relación entre ambos aspectos teniendo en cuenta la realidad que se describió previamente. En este contexto, la presente investigación se realizó en la institución pública más representativa del departamento de Puno, la Municipalidad Provincial de San Román cuya sede administrativa se encuentra en la ciudad de Juliaca en el año 2013.

En este sentido el presente trabajo de investigación es importante porque como lo afirma Peiró (1996), p. 184), la calidad de vida laboral contribuye a la mejora en el " diseño de puestos de trabajo desde las teorías clásicas de dirección y la ingeniería industrial (simplificación y especialización de tareas, control centralizado en la toma de decisiones, supervisión, uso de recompensas externas, etc.), hacia un mayor énfasis en la creación de puestos de trabajo que ofrezcan una mayor motivación intrínseca al trabajador, estrategias de diseño orientadas a la creación de grupos de trabajo autónomos, y por supuesto trabajos diseñados de tal forma que permitan la optimización del sistema social y tecnológico (p. 184).

Asimismo, la formación y el desarrollo de los trabajadores es uno de los aspectos importantes para el movimiento de la CVL, por ello considera que la formación y desarrollo personal forma parte del proceso de recursos humanos para enfatizar 
la dignidad y valor de las personas, pues todas las personas necesitan recibir información en un amplio rango de conocimientos y destrezas necesarios para el desempeño eficaz de sus tareas, pero también deben recibir formación para la toma de decisiones.

Por último, la forma de valorar el desempeño, es otro de los aportes importantes del movimiento de la CVL. Así se incluye como evaluadores a los trabajadores además de los colaboradores y supervisores. En palabras de Elera (2009), la importancia de la CVL reside en que, se logra con su aplicación empleados más saludables, con conocimientos actualizados, emocionalmente equilibrados, satisfechos con sus trabajos y su vida personal, lo cual contribuye a generar un mejor ambiente en las empresas, así mismo contribuye a la motivación de los trabajadores para alcanzar las metas propuestas.

Así también, Griffin y Moorhead (2010), afirman que los directivos de las empresas que proveen un alto grado de CVL ven los resultados positivos del trabajo de los empleados, lo cual se refleja en una mayor productividad, desarrollo de productos de alta calidad, efectividad por ende una mayor rentabilidad y cumplimiento de metas.

Por otro lado, respecto al compromiso organizacional Mowday (1982), lo considera importante pues los trabajadores más comprometidos tienen niveles más elevados de desempeño individual, lo cual ayuda a lograr las metas y los objetivos.

Por su parte, para Bowler y Brass (2006), el CO es importante porque existe mayor apoyo y aceptación de metas y los valores de la organización. Además de una mayor disposición de ejercer un esfuerzo considerable para el bien de la organización y por último generará en los colaboradores el deseo por permanecer dentro de la organización.

En el caso de Hellriegel y colbs (2009), consideran que el $\mathrm{CO}$ es importante porque solo de esta forma los colaboradores no tendrán en cuenta las fuentes menores de insatisfacción laboral y tendrán mucho tiempo de permanencia en la empresa desarrollando vínculos más profundos con la organización y sus compañeros, además permite tener actitudes positivas frente al trabajo(p.57). De forma contraria, una persona menos comprometida mantendrá una relación con la organización en términos menos personales, expresará su insatisfacción de forma abierta y no durará mucho tiempo en la organización.

Por su parte, Chiavenato (2009), identificó que el $\mathrm{CO}$ es importante porque ayuda a reducir el ausentismo, lo cual evita ciertos perjuicios para la empresa, a esto, agrega Soberanes y De la Fuente (2009:124) que "Los empleados comprometidos y calificados no requieren ser supervisados, ya que conocen la importancia y valor de integrar sus metas con la organización, porque piensan en ambas a nivel personal". Por tanto, para ellos el $\mathrm{CO}$ es importante porque tiene un impacto directo en actitudes y conductas del trabajador; como la aceptación de metas, valores y cultura, disminución de ausentismo, baja rotación de personal entre otros aspectos favorables.

Por todas estas razones expuestas, se hizo necesario realizar este estudio, a fin de ampliar los conocimientos de estos fenómenos de gestión en una institución pública; es así que se presenta un análisis de la Calidad de vida laboral y el Compromiso organizacional que se existe en los servidores públicos nombrados en la sede administrativa de la Municipalidad Provin- 
cial de San Román, para posteriormente comprobar la relación que existe entre ambas.

\section{Método}

Basados en la clasificación sugerida por Kerlinger (2002), la investigación es no experimental de corte transversal; No experimental, porque los datos se recogen tal como se encuentran en el contexto de la realidad y sin ser sometidos a ninguna manipulación durante la investigación.

$Y$ de corte transversal, conforme al sustento de Bernal (2000), quien afirma que con este tipo de diseño se obtiene información del objeto de estudio una única vez, en un momento dado. Es por ello que la recopilación y análisis de datos obtenidos en la Municipalidad Provincial de San Román se dio en un tiempo único y no repetible.

En suma, de una población de 93 personas se obtuvo una muestra probabilística de 42 encuestados entre: la Gerencia de Secretaría General, Gerencia de Administración, Gerencia de Desarrollo Urbano, Gerencia de Desarrollo Social, Gerencia de Promoción del Desarrollo Económico, Gerencia de Fiscalización y Control, Gerencia de Planeamiento y Presupuesto. Conforme a lo propuesto por Charaja (2004), se utilizó como principal técnica de recolección de datos a la encuesta y como instrumento al cuestionario auto administrado.

El mismo que comprende Aspectos Personales y Profesionales. a) La Escala de Calidad de Vida Profesional y, b) Compromiso organizacional.

El instrumento consta de: Aspectos Personales y Profesionales: Donde se persiguió identificar a la muestra a través del Sexo, Edad, Estado civil, Nivel de formación, Tiempo de servicios en la MPSR y Grupo Ocupacional, a fin de describir la Calidad de Vida Laboral y el Compromiso Organizacional del personal nombrado. 1) Escala de Calidad de Vida Profesional CVP-35 de Martín, J. y colbs. (2003): La escala está distribuida en 35 afirmaciones que permite evaluar las dimensiones de Demandas del trabajo, Motivación intrínseca, Apoyo Directivo y la percepción global de la CVL, todas ellas son puntuadas en una escala de Likert del 1 al 10 donde los valores son distribuidos de la siguiente forma: Nada: 1 y 2, Algo: 3,4 y 5, Bastante: 6,7 y 8 Mucho: 9 y 10. Para poder analizar y determinar el grado de CVL, fue necesario categorizar las medias de las respuestas obtenidas.

Dicha categorización fue tomada del baremo propuesto por Cortez, M. y Salina A. (2012). 2) Instrumento de Compromiso organizacional de Meyer y Allen (1997), con el evaluamos las dimensiones de Continuidad, Normatividad y Afectividad en 18 ítems que fueron calificados según los siguientes valores propuestos por los autores: $1=$ Totalmente en Desacuerdo. 2= Moderadamente en Desacuerdo. 3= Débilmente en Desacuerdo. $4=\mathrm{Ni}$ de Acuerdo, $\mathrm{Ni}$ en Desacuerdo. 5= Débilmente de Acuerdo. $6=$ Moderadamente de Acuerdo. $7=$ Totalmente de Acuerdo.

A fin de facilitar el análisis e interpretación de los datos obtenidos, se utilizaron los tres grados de medición definidos para esta herramienta por el baremo de Contreras, L. y Lujano, C. (1999), donde se identifican tres niveles que mostramos en la siguiente manera: Bajo (1 a 3) Medio $(3,1$ a 5,1$)$ Alto $(5,2$ a 7$)$. Los instrumentos fueron sometidos a juicio de 3 expertos para su validación con grado de doctor, maestría y especialistas en estadística. 
Asimismo, en cuanto a la Escala de Calidad de Vida Profesional CVP-35 de Martín, J. y colbs. (2004) se obtuvo, el valor del Alpha de Cronbach 0.857 muestra que todos los ítems presenta una contribución muy parecida a la consistencia global del instrumento, pues tiene una alta homogeneidad, siendo que todos sus ítems son congruentes entre sí; por tanto existe una muy buena consistencia interna del instrumento.

Luego, en cuanto al Instrumento de Compromiso organizacional de Meyer y Allen (1997), se obtuvo, el alfa de Cronbach de coeficiente de fiabilidad de 0.70 como aceptable, por ende, nuestros instrumentos han demostrado su fiabilidad y validez para esta investigación.

Finalmente, El procesamiento se dio mediante el tratamiento estadístico realizado con el Software SPSS para Windows y Microsoft Excel, de modo que inicialmente se pudieron hallar las medias de las respuestas dadas por la muestra para poder determinar el nivel de CVL y grado del $\mathrm{CO}$, en función de los aspectos personales y profesionales.

Para conocer la relación que existe entre las variables y la relación entre las dimensiones de la CVL y el CO, se sometieron los datos a la prueba estadística de Tau- $b$ de Kendall, pues es la que mejor se adapta a esta investigación y facilita el logro de los objetivos de correlación; la información procesada se representó mediante tablas estadísticas y algunas figuras con base en estos datos obtenidos.

\section{Resultados}

Considerando que los datos no provienen de una distribución normal se aplicó el modelo estadístico Tau_b de Kendall que según Nuria y Gil (1998), se usa para analizar la relación entre dos variables ordinales o rangos que tienen en consideración los empates.

Análisis de relación entre las Cargas del trabajo y el Compromiso Organizacional.

Ante las evidencias estadísticas presentadas en la Tabla $1(p=0-024$, Coeficiente de correlación $=-0,314)$. Se devela que; existe relación directa y estadísticamente significativa entre las demandas del trabajo y el compromiso organizacional del personal nombrado en la sede administrativa de la Municipalidad Provincial de San Román.

Tabla 1

Análisis de relación entre la Motivación Intrínseca y el Compromiso Organizacional.

\begin{tabular}{|c|c|c|c|c|}
\hline & & & $\begin{array}{c}\text { Cargas del } \\
\text { trabajo }\end{array}$ & $\begin{array}{c}\text { Compromiso } \\
\text { Organizacional }\end{array}$ \\
\hline \multirow{6}{*}{$\begin{array}{l}\text { Tau_b de } \\
\text { Kendall }\end{array}$} & \multirow[t]{3}{*}{ Cargas del trabajo } & Coeficiente de correlación & 1,000 &,$- 314^{*}$ \\
\hline & & Sig. (bilateral) & . & ,024 \\
\hline & & $\mathrm{N}$ & 42 & 42 \\
\hline & \multirow{3}{*}{$\begin{array}{l}\text { Compromiso } \\
\text { organizacional }\end{array}$} & Coeficiente de correlación &,$- 314^{*}$ & 1,000 \\
\hline & & Sig. (bilateral) & 024 & \\
\hline & & $\mathrm{N}$ & 42 & 42 \\
\hline
\end{tabular}

*. La correlación es estadísticamente significativa al nivel 0,05 (bilateral). 
Según los resultados obtenidos en el análisis de relación entre la motivación intrínseca y el compromiso organizacional de los servidores públicos nombrados en la sede de la MPSR, los cuales se resumen en la Tabla 2, en la cual se obtuvo un valor $p=0.343$ y un coeficiente de corre- lación de 0.131 , lo que indica que No existe relación directa y significativa entre la Motivación intrínseca y el Compromiso organizacional del personal nombrado en la sede administrativa de la Municipalidad Provincial de San Román.

Tabla 2

Análisis de relación entre el Apoyo Directivo y el Compromiso Organizacional.

\begin{tabular}{lllll}
\hline & & & $\begin{array}{l}\text { Motivación } \\
\text { intrínseca }\end{array}$ & $\begin{array}{l}\text { Compromiso } \\
\text { organizacional }\end{array}$ \\
\hline & $\begin{array}{l}\text { Motivación } \\
\text { intrínseca }\end{array}$ & Coeficiente de correlación & 1,000 &, 132 \\
Tau_b de & & Sig. (bilateral) &. &, 343 \\
Kendall & & $N$ & 42 & 42 \\
& & Coeficiente de correlación &, 132 & 1,000 \\
& Compromiso & Sig. (bilateral) &, 343 &. \\
& organizacional & $\mathrm{N}$ & 42 & 42 \\
\hline
\end{tabular}

Al respecto, se realizó la prueba Tau b- de Kendall tal y como se presenta en la Tabla 3. Para hallar la relación existente entre el Apoyo directivo y el Compromiso Organizacional, cuyos resultados fueron $p=$ 0.631 con un Coeficiente de correlación
$=0.067$, por tanto, no existe relación directa y estadísticamente significativa entre el Apoyo directivo y el Compromiso organizacional del personal nombrado en la sede administrativa de la Municipalidad Provincial de San Román.

Tabla 3

Análisis de relación entre la Calidad de Vida Laboral y el Compromiso Organizacional.

\begin{tabular}{|c|c|c|c|c|}
\hline & & & $\begin{array}{c}\text { Apoyo } \\
\text { directivo }\end{array}$ & $\begin{array}{c}\text { Compromiso } \\
\text { organizacional }\end{array}$ \\
\hline \multirow[t]{6}{*}{ Tau_b de Kendall } & Apoyo directivo & Coeficiente de correlación & 1,000 & ,067 \\
\hline & & Sig. (bilateral) & $\cdot$ & ,631 \\
\hline & & $\mathrm{N}$ & 42 & 42 \\
\hline & Compromiso & Coeficiente de correlación & ,067 & 1,000 \\
\hline & organizacional & Sig. (bilateral) & 631 & \\
\hline & & $\mathrm{N}$ & 42 & 42 \\
\hline
\end{tabular}

*. La correlación es estadísticamente significativa al nivel 0,05 (bilateral).

En la Tabla 4. se muestra el resultado de la prueba Tau- $b$ de Kebdall para los datos agrupados de la Calidad de Vida laboral y el Compromiso organizacional, allí se visibiliza una correlación de 0.035 y un valor de significancia de $p=0.801$, valores que indican que No existe relación directa y significativa entre la Calidad de vida laboral y el Compromiso organizacional del personal nombrado en la sede administrativa de la Municipalidad Provincial de San Román. 
Tabla 4

\begin{tabular}{|c|c|c|c|c|}
\hline & & & $\begin{array}{l}\text { Calidad de } \\
\text { vida laboral }\end{array}$ & $\begin{array}{c}\text { Compromiso } \\
\text { organizacional } \\
\end{array}$ \\
\hline \multirow[t]{6}{*}{ Tau_b de Kendall } & \multirow{3}{*}{$\begin{array}{l}\text { Calidad de vida } \\
\text { laboral }\end{array}$} & Coeficiente de correlación & 1,000 & ,036 \\
\hline & & Sig. (bilateral) & & ,801 \\
\hline & & $\mathrm{N}$ & 42 & 42 \\
\hline & \multirow{3}{*}{$\begin{array}{l}\text { Compromiso } \\
\text { organizacional }\end{array}$} & Coeficiente de correlación & ,036 & 1,000 \\
\hline & & Sig. (bilateral) & ,801 & . \\
\hline & & $\mathrm{N}$ & 42 & 42 \\
\hline
\end{tabular}

\section{Discusión}

Análisis de relación entre las Cargas del trabajo y el Compromiso Organizacional.

Ante las evidencias estadísticas presentadas en la Tabla 1 ( $p=0-024$, Coeficiente de correlación $=-0,314)$, es evidente que la correlación obtenida fue de 0,314 , lo que indica que existe una correlación negativa y baja, por tanto se presenta una asociación inversa, significando ello que si las Cargas de trabajo se incrementan el Compromiso Organizacional disminuye un poco y viceversa. Dicho razonamiento, pareciera ser lógico, pues si los mandos superiores de la MPSR incrementan la presión por la calidad o cantidad del trabajo, crecen las incomodidades físicas, las responsabilidades, los conflictos, entre otras cargas laborales.

Al respecto, Casas (2002), afirma que esta sobrecarga de trabajo está asociada a la insatisfacción y el estrés laboral; por tanto el personal nombrado se sentiría insatisfecho en su trabajo y por esta razón disminuiría un poco el compromiso hacia su institución.

Análisis de relación entre la Motivación Intrínseca y el Compromiso Organizacional.

A propósito de este análisis, con el fin de evidenciar la relación entre la Motivación intrínseca y el Compromiso organizacio- nal de los servidores públicos nombrados en la sede de la MPSR, los cuales se resumen en la Tabla 2, en la cual se obtuvo un valor $p=0.343$ y un coeficiente de correlación de 0.131 , es notable que No existe relación directa y estadísticamente significativa entre la Motivación intrínseca y el Compromiso organizacional del personal nombrado en la sede administrativa de la Municipalidad Provincial de San Román.

Ahora bien, luego de sometidos los datos a la prueba estadística de Tau- $b$ de Kendall, se puede observar en la Tabla 3 un valor calculado de $p=0.343$ a un nivel de significativa de 0,05 (bilateral), siguiendo la regla $p>\alpha$, afirmándose que No existe relación significativa entre la Motivación intrínseca y el Compromiso organizacional.

Análisis de relación entre el Apoyo Directivo y el Compromiso Organizacional.

De igual forma, se realizó la prueba Tau b- de Kendall para hallar la relación existente entre el Apoyo directivo y el Compromiso Organizacional, cuyos resultados halados fueron $p=0.631$ y Coeficiente de correlación $=0.067$, por tanto, se evidencia que No existe relación directa y significativa entre el Apoyo directivo y el Compromiso organizacional del personal nombrado en la sede administrativa de la Municipalidad Provincial de San Román. 
En consecuencia, no existe relación estadísticamente significativa entre la dimensión de la CVL Apoyo directivo y el Compromiso organizacional de forma conjunta, pues se halló un valor calculado de $p=0.631$ a un nivel de significatividad de 0,05 bilateral $(p>\alpha)$.

Análisis de relación entre la Calidad de Vida Laboral y el Compromiso Organizacional.

Es de hacer notar la realización de la prueba Tau- $b$ de Kebdall para los datos agrupados de la Calidad de Vida laboral y el Compromiso organizacional, los resultados se evidencian en la Tabla 4, allí se halla una correlación de 0.035 y un valor de significancia de $p=0.801$, valores que indican que No existe relación directa y significativa entre la Calidad de vida laboral y el Compromiso organizacional del personal nombrado en la sede administrativa de la Municipalidad Provincial de San Román.

Ahora bien, como se pudo observar en los resultados previos a propósito de la prueba estadística de Tau_b de Kendall se determina que la Calidad de Vida laboral no presenta relación estadísticamente significativa con el Compromiso Organizacional $(p=0.801)$.

Entonces, al tomar en cuenta el coeficiente de correlación 0,035 ; se puede afirmar que la correlación es muy débil prácticamente nula, por lo que no necesariamente una mejor Calidad de Vida Laboral, da como resultado un grado más alto de Compromiso Organizacional.

Dicho resultado, se sustenta con palabras de Cohen (1988), que en los temas que conciernen a la conducta humana, como lo es el Compromiso Organizacional, las correlaciones frecuentemente suelen ser bajas y no por ello menos importantes.

Sin embargo, para Morales (2008), el coeficiente hallado puede aportar información útil para pensar por qué no existe una relación apreciable en la cual cabría esperarla, por ello es necesario hacer algunas consideraciones respecto de las razones de este hallazgo. En este sentido, se considera pertinente tener en cuenta ciertos aspectos que permitirán en cierto modo comprender este fenómeno.

De modo que, en primera instancia, se ha de tener en cuenta que la investigación se realizó en una institución pública: la Municipalidad Provincial de San Román, que es considerada la más grande e importante en términos de presupuesto público asignado, y en función al tamaño de la población a la que brinda sus servicios, una gran estructura jerárquica, entre otros aspectos; que sin lugar a duda la hace una organización compleja en términos de dirección y más aún cuando se busca la mejora de la CVL o se persigue el incremento del grado de compromiso, pues como es sabido, ambos dependen de muchos factores que pueden favorecerla o hacer que decrezca.

\section{Conclusión}

1. Es notable que no existe relación estadísticamente significativa entre la Calidad de Vida Laboral percibida y el Compromiso Organizacional del personal nombrado en la sede administrativa de la Municipalidad Provincial de San Román; por tanto las dimensiones que componen la Calidad de Vida Laboral en una institución pública, no influyen de manera relevante en el compromiso que el personal nombrado tiene hacia su institución, 
2. pues ellos otorgan mayor importancia a otros aspectos que determinan su compromiso, como el logro de la estabilidad laboral a través del nombramiento, más que a la Calidad de Vida Laboral.

3. La relación entre las Cargas del trabajo y el Compromiso Organizacional del personal nombrado en la sede administrativa de la Municipalidad Provincial de San Román es directa y estadísticamente significativa en orden negativo, lo que indica que si se incrementaran las Cargas del trabajo se vería disminuido el Compromiso Organizacional y viceversa.

4. El estudio demostró que existe una relación débil entre la Motivación intrínseca y el Compromiso organizacional del personal nombrado, pues la motivación intrínseca implica diferentes procesos internos que no necesariamente dependen de las acciones emprendidas por parte de la dirección, los cuales no tienen ninguna relación con el grado de compromiso hacia su institución.

5. La relación entre el Apoyo Directivo y el Compromiso Organizacional es inexistente, por tanto, según la muestra investigada, por más que los gerentes, subgerentes y jefes de las diferentes áreas, se enfoquen en incrementar el nivel de apoyo hacia el personal nombrado que tienen a su mando, esto no disminuirá ni incrementará el grado de compromiso que poseen los mismos hacia su institución.

\section{Recomendaciones}

1. Siendo que no existe relación entre las variables de la Calidad de Vida Laboral y el Compromiso Organizacional, se invita a tener en consideración para futuras investigaciones, correlacionar otras variables que pudieran tener mayor relación con la Calidad de Vida Laboral y el Compromiso Organizacional en una institución pública.

2. A pesar de que no existe relación entre las variables, se sabe según lo establecido en el marco teórico de esta investigación que la Calidad de Vida laboral y el Compromiso Organizacional reportan múltiples beneficios para la entidad en la que se trabaja, para el personal nombrado y como consecuencia para los usuarios, es por ello que se exhorta iniciar la implementación de un plan de mejora para la Calidad de Vida Laboral, y a su vez desarrollar programas conjuntos de recursos humanos los cuales logren incrementar el grado de Compromiso Organizacional.

3. Siendo que las Cargas laborales tienen relación negativa con el Compromiso Organizacional,se invita a la Sub Gerencia de personal, tomar las acciones correctivas $y$ preventivas respecto a la distribución de las cargas del trabajo que tiene el personal nombrado, tales como evitar presiones innecesarias y exageradas en el trabajo, proporcionar cargas de trabajo adecuadas 
que vayan acorde con las responsabilidades de su cargo, a fin de que no se interfiera con su vida personal fuera de la institución. Por último, identificar las incomodidades físicas que dan mayor malestar a los colaboradores en las diferentes Gerencias y Sub Gerencias para incluir en el Plan Operativo Anual de Recursos Humanos es vital, es decir manejar un presupuesto exclusivo para las mejoras correspondientes en los aspectos identificados, pues de no ser así el grado de Compromiso se verá disminuido.
4. Se recomienda desarrollar investigaciones que profundicen los estudios respecto a la Calidad de Vida Laboral y el Compromiso organizacional en otras circunstancias y en otras instituciones públicas, lo cual permitirá realizar comparaciones de resultados y futuras generalizaciones con respecto a esta temática, de ese modo se podrán crear vínculos con un área de investigación que cada vez gana mayor importancia en el medio administrativo. 


\section{Referencias}

Bernal, C. (2000). Metodología de la Investigación para Administración y Economía. Colombia: Pearson Educación. Pp.110-114.

Bowler, M. y Brass, D. (2006).Relational correlates of interpersonal citizenship behavior: A social network perspective. Journal of AppliedPsychology.(13), 7082.

Charaja, F. (2004). Investigación Científica (2da ed.). Perú: Nuevo Mundo. P.25.

Chiavenato, I. (2009 a). Comportamiento Organizacional: La dinámica del éxito en las organizaciones (2da ed.). México: McGraw Hill. pp. 6, 12.

Casas J., Repullo J., Lorenzo S., Cañas J. (2002). Dimensiones y medición de la calidad de vida laboral en profesionales sanitarios. Revista de Administración Sanitaria, 6 (23).Pp527-44.

Contreras, L., Lujano, C. (1999). El Compromiso Organizacional en trabajadores accionistas. Tesis de Grado en Relaciones industriales, Universidad Andrés Bello, Venezuela. Recuperado el 22 de enero del 2013 enhttp://www.ucab.edu.ve/tesisdigitalizadas2/orderby/ths_author/sort/a sc/ths_year/1999/ths_grade/escuela-deciencias-sociales.html

Córtez, M. y Salina, A. (2012). Calidad de Vida Profesional en la Enfermeras 2012. Informe de Investigación en la Universidad Nacional de Córdoba. pp 29 y 30 .

Elera, C. (2009). Administración y Gestión del potencial Humano. Un enfoque a la gestión de la Administración de los recursos Humanos en el Perú (1ra ed.). Lima, Perú: Editorial de la Universidad de San Martín de Porres. Pp 73, 547.
Griffin, R. y Moorhead, G. (2010). Comportamiento Organizacional (9na ed.). Australia: CengageLearning. pp 70, 505,506

Hellriegel, D. y Slocum, J.(2009). Comportamiento Organizacional (12ava ed.) . México: CengageLearning Editores. P.57.

Kerlinger, F. (2002). Investigación del comportamiento: métodos de investigación en Ciencias Sociales. México, D.F.: Mc Graw-Hill. 68- 137.

Martín, J., Cortéz, J., Morente, M., Caboblanco, M., Garijo, J. y Rodríguez, A. (2003). Características métricas del Cuestionario de Calidad de Vida Profesional (CVP-35). Revista Gaceta Sanitaria, Barcelona. 18 (2). 18. p 129136

Meyer, J., Allen, N. (1997). Teoría del Compromiso en el lugar de trabajo: investigación y aplicación. Londres: Sage Publications. Pp. 27-173.

Morales, P. (2008) Estadística aplicada a las Ciencias Sociales.Madrid España Edit. De la Universidad Pontificia Comillas. Pp 79, 314-317.

Mowday, R, Porter, L., Steers, R. (1982). Vínculos de los empleados de la organización: La psicología del compromiso, ausentismo y rotación. Nueva York: AcademicPress. Pp.36-59.

Peiró, José. (1996). Tratado de Psicología del trabajo: La actividad laboral en su contexto.España: Síntesis S.A. pp. 38,163,164, 184.

Reyes, J. (2009). Fortalecimiento de Capacidades de Funcionarios y Servidores Públicos en el Perú. Recuperado el 13 de enero, de http://www.arcastilla.org/articulos/desarr ollo_de_capacidades.ppt pp.13-17. 
Robbins, S. y Judge, T. (2009). Comportamiento Organizacional. (13 ava ed.)México: Pearson Prentice Hall. Pp. $10,24,79$

Soberanes, L. y De la Fuente, A. (2009). El Clima y el compromiso organizacional en las organizaciones. Revista internacional la Nueva Gestión Organizacional 5 (9) p.124. Recuperado el 21 de Mayo del 2013 en http://www.google.com.pe/url?sa=t\&rct=j $\& q=\&$ esrc $=s \&$ frm $=1 \&$ source $=$ web $\& c d=5$ \&sqi=2\&ved $=0$ CEwQFjAE\&url $=$ http $\% 3 A$ $\% 2 \mathrm{~F} \% 2 \mathrm{Fwww}$.tdx.cat\%2Fbitstream\%2F 10803\%2F5431\%2F1\%2Fjna1de1.pdf\& ei=TguJUe_FDePMiQLh4IC4CA\&usg=A FQjCNHWPtu0QcSnVX3XWJM7ZuwKV mfkhw\&sig2=xI_d_f1qvPNByquj8fMCSw
Stoner, J.,Freeman, E. y Gilbert, D. (1996). Administración. (13ava ed.). México: Prentice Hall Hispanoamericana. S.A. p.36.

Ugarte, M, Celle, M y Sotomarino, N. (2011). Gestión Pública (1ra ed.). Lima, Perú: Consorcio de investigación económica y social. Pp.17,18, 41-47. 